\title{
Jan Hemming, Methoden der Erforschung populärer Musik (Systematische Musikwissenschaft), Wiesbaden: Springer 2016, https://doi.org/10.1007/978-3-658-11496-1
}

Schlagworte/Keywords: Lehrbuch; Methoden; methods; Paradigmen; paradigms; Popular Music Studies; populäre Musik; textbook; Theorie; theory

Seit ihrer akademischen Etablierung in den frühen 1980er Jahren steht die Erforschung der populären Musik auf einem interdisziplinären Fundament. Unter dem heute gebräuchlichen Anglizismus Popular Music Studies versammeln sich u. a. musikwissenschaftliche, literaturwissenschaftliche, kulturwissenschaftliche, soziologische, ethnologische und medienwissenschaftliche Forschungszugänge. Das Feld der Popmusikforschung ist breit gefächert. Vor diesem Hintergrund stellt das Verfassen eines Buches, das sich methodischen Fragen widmet, eine große Herausforderung dar. Die Monografie Methoden der Erforschung populärer Musik (2016) von Jan Hemming, Professor für Systematische Musikwissenschaft an der Universität Kassel, darf von daher als ein sehr mutiges Projekt verstanden werden. Das 534 Seiten starke Buch ist beim Springer-Verlag in der Reihe "Systematische Musikwissenschaft" erschienen. Nach Begabung und Selbstkonzept. Eine qualitative Studie unter semiprofessionellen Musikern in Rock und Pop (2002) und Improkomposition. Buch I der imaginären Geometrie zur Anwendung in der Klavierimprovisation (2013, in Ko-Autorenschaft mit Andre von Melöchin) handelt es sich um Hemmings dritte Monografie.

Sofort beim ersten Einblick in das Inhaltsverzeichnis mit seinen elf Kapiteln wird klar, dass hier eine äußerst ambitionierte Arbeit vorgelegt wurde, welche den genannten vielfältigen Forschungszugängen gerecht werden möchte. Gleich zu Beginn der Einleitung weist Hemming auf die Interdisziplinarität des Forschungsfeldes hin und formuliert den Grundgedanken des Buchs, »existierende Zugänge durch Anwendungsbeispiele zu veranschaulichen und damit auch Anregungen zum >Weiterforschen zu geben« (13). Ferner wird ange- führt, dass solch ein Überblick in Buchform bisher nicht existiere oder nur ausschnittsweise in Sammelbänden oder in weniger musikwissenschaftlich fokussierten Monografien vorgelegt worden sei. Dabei ist das von Hemming in einer Fußnote genannte Studying Popular Music von Richard Middleton aus dem Jahr 1990 sicherlich als erstes und wohl bisher einziges Standardwerk anzusehen. ${ }^{1}$

Hemming betont den Lehrbuchcharakter seiner Monografie, deren Ziel es sei, Perspektiven aufzuzeigen. Er will es »dem Leser überlassen, ob etwa eine Orientierung am musikalischen Text gegenüber einer Beschäftigung mit dem soziokulturellen Kontext zu bevorzugen sei, oder ob einer theoretischen gegenüber einer empirischen Vorgehensweise der Vorrang eingeräumt werden soll« (14). Diese grundsätzliche Offenheit gegenüber verschiedenen methodischen Zugängen ist zu begrüßen. Gleichzeitig führt dies aber auch zu einem großen Manko des Buches, das sich sehr oft in den Details seiner vielen Einschübe verliert, ohne dabei systematisch und kritisch verschiedene Paradigmen zu sortieren und zu diskutieren. Auch verwundert in diesem Einleitungskapitel, dass Hemming das »komplexe Problem« (14) der Definition populärer Musik an das Ende des Buches (Kapitel 11) verlagert. Selbst wenn die verschiedenen Kapitel nicht aufeinander aufbauen und unabhängig voneinander gelesen werden können, so wäre doch von einem Lehrbuch zu erwarten gewesen, dass die Definition oder Eingrenzung des Forschungsgegenstands am Anfang steht. Jedes der Kapitel ist mit Grafiken, Bildern und Notenbeispielen verse-

$1 \quad$ Middleton 1990 
hen. Im Anhang der einzelnen Kapitel findet sich ein Literaturverzeichnis der verwendeten und weiterführenden Quellen.

In Kapitel 1 »Musikwissenschaft und populäre Musik: Ein theoretisches Rahmenmodell« arbeitet Hemming die Adler'sche Unterscheidung zwischen Historischer und Systematischer Musikwissenschaft kritisch auf und verortet dabei wesentliche Missverständnisse, die in diesen beiden Teildisziplinen in Bezug auf die Erforschung populärer Musik entstanden seien. Populäre Musik könne nicht allein als Text analysiert werden. Der Einbezug soziokultureller Phänomene ist, wie Hemming herausstellt, für die Erforschung populärer Musik unumgänglich, dennoch, so darf angemerkt werden, setzt der Autor damit eine Dichotomie zwischen Text und Kontext voraus, die bereits häufiger in der Popmusikforschung problematisiert wurde. ${ }^{2}$ Das Modell von Wechselwirkungen zweier ontologisch getrennter Ebenen ist bei jüngeren Ansätzen, insbesondere bei den Sound Studies oder bei praxeologischen und medienwissenschaftlichen Zugängen, die Hemming im ganzen Buch eher vernachlässigt, angefochten worden.

In den folgenden Abschnitten führt Hemming stark komprimiert in die marxistische Kulturtheorie ein - mit Bezug auf Marx, Kritische Theorie und Cultural Studies (35-44). Damit benennt er zentrale Forschungsprobleme der Popular Music Studies (beispielsweise das angesprochene Text-Kontext-Problem oder das Problem eines normativen Werkbegriffs). Stutzig macht jedoch ein angehängter zweiseitiger Abschnitt (44f.) zu "Empirische[n] Verfahren" und "Transdisziplinarität», die verglichen mit den Abschnitten über Kritische Theorie und Cultural Studies nicht konkret ausgeführt werden. Die »Brücken«, die hier zwischen marxistischen Kulturtheorien und empirischen Verfahren »in Sicht« (44) seien, werden nur in einer Fußnote mit Verweis auf weitere Literatur abgehandelt und nicht weiter vertieft.

Kapitel 2 »Technologie und Produktion" platziert zwei Begriffe als Überschrift, um die herum sich zentrale Felder der Popmusikforschung entwickelt haben. Die Ausführungen

2 Vgl. Wicke 2003. zum akustischen Aufnahmezeitalter (ca. 18901925) und zu den Pionierjahren des elektrischen Aufnahmezeitalters (ca. 1925-45) fallen sehr knapp aus (51f.). Den Technikentwicklungen der ersten Hälfte des 20. Jahrhunderts wird kein eigener Abschnitt zugestanden. Hemming springt stattdessen direkt zur "Session Aufnahme« (52-57) der Nachkriegszeit und bespricht ausführlich die Technologie des Tonbandes. Es folgen u. a. Abschnitte zur »Arbeitsteilige(n) Studioproduktion « (57-59) und zu »Homerecording-Studio/Audio-Workstation" (60-66). Statt verschiedene Forschungsparadigmen einander gegenüberzustellen, geht Hemming sehr eklektisch vor und zitiert eine begrenzte Auswahl an Veröffentlichungen. Das ganze Kapitel liest sich eher wie eine kurze Technikgeschichte der populären Musik denn als eine Diskussion von unterschiedlichen Methoden zu ihrer Erforschung, wie sie etwa mit medienwissenschaftlichen bzw. medientechnischen Zugängen vorgelegt werden könnte (etwa einer Medienarchäologie oder einer Akteur-NetzwerkTheorie $^{3}$ ). Der inzwischen beträchtlich gewachsene Forschungszweig einer Musicology of Record Production « wird nur in wenigen Zeilen erwähnt (65), anstatt hier ausführlicher auf zugehörige Veröffentlichungen und deren Befunde einzugehen (der Verweis auf entsprechende Publikationen findet sich nur als »weiterführende Literatur « im Anhang des Kapitels). ${ }^{4}$

Kapitel 3 »Textuelle Analyse« beginnt mit der Feststellung, dass eine allgemeine Musiktheorie für die populäre Musik nicht existiert. Populäre Musikpraxis fußt wesentlich auf Informalität, nur sehr selten liegt dem Musikmachen und der Aneignung von popmusikalischen Kompetenzen ein explizites Regelwerk zu Grunde (77f.). Hemming plädiert, im Anschluss an eine bereits jahrelang geführte Debatte in den Popular Music Studies, für popmusikspezifische Analysezugänge. Auch wahrnehmungspsychologische Aspekte, die in der Systematischen Musikwissenschaft erforscht werden, könnten hier weiterhelfen (79-81). Es gebe nicht ein >richtiges` oder ein sfalsches Musikhören, stattdessen müssten multiple $\mathrm{Zu}$ -

3 Vgl. Schröter/Volmar 2013; Butler 2014.

4 Vgl. Zak 2001; Zagorski-Thomas 2014. 
gänge auch bei der Analyse legitim sein. Formuliert Hemming damit im Kern eine produktive und sinnvolle Annäherungsweise für die Analyse populärer Musikformen, so wird im Verlauf des Kapitels nicht auf einschlägige Monografien hierzu eingegangen. ${ }^{5}$ Stattdessen geht Hemming alle straditionellens Parameter der Musiktheorie durch und führt sehr grundsätzlich (bei Pythagoras beginnend!) in Theorien der Tonarten, Skalen, Melodie, Harmonik, Metrum, Takt, Rhythmus, Groove, Songtext, Sound und Textur, Form und Formate ein. Dabei entwickelt der Autor durchaus einleuchtende Gedanken zu popmusikspezifischen Analysezugängen: So kenne die populäre Musik keine Funktionsharmonik, stattdessen gehe es u. a. um Akkordfolgen und -zusammensetzungen, die durch die Spielbarkeit eines Instruments ermöglicht werden (etwa Power-Chords auf der Gitarre). Auch lebe populäre Musik von genuinen Melodiegestaltungen, die der klassischromantischen Musikpraxis eher fremd seien (z. B. blue notes, Anschleifungen). Verwundert ist man allerdings über die Delegitimierung der inzwischen einflussreichen Sound Studies als "modischen Studiengang" (114), die Sound bzw. Textur nicht sinnvoll musikanalytisch erschließen würden. Diese pauschale Behauptung bleibt unbegründet. Hemming vermeidet an dieser Stelle eine Diskussion von Veröffentlichungen aus dem Bereich der Sound Studies.

Nach der Einführung von musiktheoretischen Grundbegriffen folgt eine Systematisierung von Analyseverfahren (118-147). Die lose Aneinanderreihung von einzelnen Beispielen (The Beatles, Keith Jarret, Jeannette Biedermann bis hin zur Rechtsrockband Nahkampf) hilft nicht sonderlich, um einen Überblick zu unterschiedlichen Verfahren der Notentextanalyse und grafischen Analyse zu gewinnen. Das Kapitel endet mit einem eigens von Hemming und Andre von Melöchin entwickelten grafischen Verfahren zur Darstellung musikalischer

5 Vgl. etwa Brackett 1995; Hawkins 2002; Moore 2012. Lediglich in der »weiterführenden Literatur « im Anhang des Kapitels wird am Rande auf andere Veröffentlichungen zur Popmusikanalyse verwiesen: Elflein 2010; Rappe 2010; Helms/Phleps 2011.
Verläufe mit Hilfe von geometrischen Figuren (144-147). Der Mehrwert dieses interessanten, obschon sehr abstrakten Darstellungsmodells, verglichen mit herkömmlicher Notenschrift oder grafischen Verlaufstabellen, erschließt sich in dieser kurzen Ausführung leider nicht.

Kapitel 4 "Semiotische Analyse" führt in die Zeichentheorie (speziell in die von Charles Sanders Peirce) ein (160-170). Diese wird auf die Analyse populärer Musik übertragen, wobei fast ausschließlich an die Arbeiten von Philip Tagg aus den späten 1970er und frühen 1980er Jahren angeknüpft wird (170-179). ${ }^{6}$ Sodann folgen zwei Anwendungsbeispiele einer an Tagg angelehnten semiotischen Analyse zu Musik in "Werbespots der ARD Fernsehlotterie« (179-185) und zur »Rechten Musik» (185193). Hemming wendet Taggs Konzept des musemes an. Musemes sind kleine bedeutungstragende Einheiten einer musikalischen Struktur (charakteristische Motive, spezifische Klangtexturen, Instrumentierungen o. ä.), die mit kulturellen Bedeutungen aufgeladen sind und damit Verweischarakter haben. Auf dieser Grundlage arbeitet Hemming bei seiner Analyse der Werbespots u. a. strukturelle Entsprechungen zwischen Bild- und Musiksequenzen heraus und kommt bei seiner Analyse von typischen Sounds rechter Musik zur Feststellung, dass hier auf Texturen zurückgegriffen wird, die kulturell mit »Energiegeladenheit, Motorik und auch Aggression « (191) assoziiert sind. Hemming stellt am Schluss des Kapitels die Überlegung an, dass es denkbar wäre, Musik auch auf Bedeutungen hin zu analysieren, die jenseits einer Zeichenhaftigkeit liegen und womöglich in Körperlichkeit und Affektstrukturen zu suchen sind. Dem ist hinzuzufügen, dass Julia Kristeva und Roland Barthes bereits die Grundzüge einer solchen alternativen Semiologie entwickelt haben, die über Verkörperungen und Materialität funktioniert (Barthes spricht von der "Rauheit der Stimme» und Kristeva unterscheidet zwischen "Genotext" und »Phänotext«). ${ }^{7}$ Diese Semiologie wurde bereits als Grundlage für die Entwicklung musiktheoretischer Modelle und Musikanalysen herangezo-

$6 \quad$ Tagg 1982; Tagg 2000; Tagg 2001.

7 Vgl. Kristeva 2010; Barthes 2013. 
gen. ${ }^{8}$ Einen Überblick und eine Gegenüberstellung von unterschiedlichen musiksemiotischen Modellen oder Alternativen zu Taggs Ansatz gibt Hemming allerdings nicht.

In Kapitel 5 »Gender Studies und Performativität« stellt Hemming Ansätze zur Geschlechterforschung im Kontext der Popmusikforschung vor. Aus der inzwischen beträchtlich gewachsenen Literatur zu Gender und Musik verweist der Autor primär auf Arbeiten, die nicht der Popmusikforschung im engeren Sinn, sondern der Historischen Musikwissenschaft zugerechnet werden müssen (z. B. Susan McClarys Feminine Endings). Etliche Reader, Monografien und Aufsätze zu Geschlecht und Queerness in populärer Musik wären hier heranzuziehen gewesen, ${ }^{9}$ wohingegen Hemming vor allem Beispiele aus der Kunstmusik anführt (Chopin, Schumann, Beethoven). Der Abschnitt "Anwendungsbeispiel: Musik und Körper « hat dann zunächst wenig mit vergeschlechtlichter Körperlichkeit zu tun, wie sie etwa durch Klangpraktiken untersucht werden könnte, und geht stattdessen - in einem der vielen Einschübe dieser Arbeit - auf physiologische Wirkungen von Klang ein. Hemming fragt nach der körperlichen Empfindung von Gitarrenverzerrung, nutzt diesen Einschub aber eher, um ausführlich über die Entwicklungen und Bauweisen von Gitarrenverstärkern zu referieren. Dabei verliert er den eigentlichen Fokus auf die Frage nach Geschlecht und Körperlichkeit im Klangerleben. Im Anschluss wird somit nicht klar, wie der Autor das Klangerleben mit kulturwissenschaftlichen Theorien des vergeschlechtlichten Körpers (u. a. Semiotik und Performativität nach Judith Butler) verknüpfen möchte, sodass ein produktiver methodischer Zugriff auf das Verhältnis von Musik und Körper nicht zu erkennen ist. Die bereits angesprochenen Theorien von Kristeva und Barthes sowie Veröffentlichungen aus dem Bereich der

8 Zu Anwendungen auf Musiktheorie/Musikanalyse vgl. Shepherd/Wicke 1997 und JarmanIvens 2011.

9 Vgl. etwa Whiteley 1997; Whiteley/Rycenga 2006; Jarman-Ivens 2007; Heesch/Hornberger 2015.
Sound Studies, ${ }^{10}$ die Hemming nicht zur Kenntnis nimmt, hätten für die Diskussion um vergeschlechtlichte Körperlichkeit und Klang fruchtbar gemacht werden können. ${ }^{11}$ So kann Hemming in seinem Fazit (227f.) keinen überzeugenden methodischen Zugriff vorlegen.

Kapitel 6 »Empirische Forschung « führt ausführlich in die quantitativen (Fragebogen) und qualitativen (Interview) Methoden der Sozialforschung ein. Auffällig ist die Diskrepanz im Umfang zwischen diesem Kapitel (87 Seiten) und dem vorangegangenen Kapitel »Gender Studies und Performativität« (35 Seiten). Man merkt, dass Hemming als Systematischer Musikwissenschaftler in diesem Feld zu Hause ist. Das Kapitel beginnt mit allgemeinen Ausführungen zu quantitativen und qualitativen Verfahren ohne besonderen Popmusikbezug. Es liest sich entsprechend eher wie eine grundsätzliche Einführung zu empirischen Begriffen und Methoden. Beim Anwendungsbeispiel "Zur Phänomenologie des Ohrwurms" (265287) wird nicht klar, warum denn genau der Ohrwurm als typisches popmusikspezifisches Phänomen zu erforschen ist. Das zweite lange Beispiel beschäftigt sich mit der Frage "Wie erleben Musiker ihr eigenes Konzert? (287315). Mit diesen Anwendungsbeispielen werden ausführlich konkrete Forschungen und deren Befunde vorgestellt. Einen umfassenden Überblick zur Programmatik einer empirischen Popmusikforschung gewinnt man jedoch nicht.

Kapitel 7 »Kontextuelle Analyse« beginnt mit einer Darstellung des Text-KontextProblems. Unter Berufung auf den Musikanthropologen Steven Feld merkt Hemming an, dass es bei einer kontextuellen Analyse darum ginge "sound as social structure" (323) zu analysieren. Zunächst werden soziologische und psychologische Konzepte eingeführt (Aspekte der Sozialisation, das 'Big Five-Modell der Persönlichkeitsforschung, Lerntheorien), um dann in einem eineinhalbseitigen Einschub über »DJ-Techniken im Hip-Hop« zu sprechen, die als »Musterbeispiel für selbsterfundene und

10 Vgl. etwa Henriques 2011.

11 Jüngst hat L. J. Müller eine Monografie vorgelegt (Müller 2018), die solch eine Theorie zu Körper, Geschlecht und Klang aufstellt. 
selbsterworbene Fähigkeiten « (333) gelten sollen. Es folgt ein kurzer Abschnitt, in dem die Begriffe der Homologie und Subkultur aus den Cultural Studies definiert werden. Über einen noch kürzeren Abschnitt - der eher als Abriss bezeichnet werden muss - zu Ethnographien von Musikszenen und -netzwerken gelangt Hemming zu drei längeren Anwendungsbeispielen: »Persönlichkeit und Verhalten der Fans von Hard Rock, Punk und Gangsta Rap«, »Ansätze einer Metastudie zu Musik und Aggression « und "Bleibt man ein Leben lang mit der Musik verbunden, die man mit Anfang 20 kennengelernt hat? " (339-368). Unter diesen Überschriften diskutiert und präsentiert Hemming Ergebnisse verschiedener Forschungen (inklusive seiner eigenen). Grundsätzliche Methodenfragen spielen dabei eher eine nachgeordnete Rolle. Das Kapitel schließt letztlich, ohne dass der oder die Leser*in etwas über die Vor- oder Nachteile der einzelnen Forschungsansätze (empirische Sozialforschung, Cultural Studies, ethnographische Methoden) erfährt. Ein Fazit fehlt.

Kapitel 8 »Ökonomische Analyse: Musikindustrie und Urheberrecht« ist als Einführung in Methoden zur Untersuchung von industriellen und musikrechtlichen Institutionen angelegt. Dies geschieht in Bezug auf das Mediamorphose-Modell Kurt Blaukopfs. Hier werden also sinnvollerweise die Vermarktung und Verrechtlichung von populärer Musik an Speichermedien, also die Artefaktkultur, gekoppelt. Aufgrund des exklusiven Zugriffs auf die musikrechtlichen und musikökonomischen Institutionen über Blaukopfs Mediamorphose-Modell fehlt aber auch hier eine Gegenüberstellung unterschiedlicher Paradigmen. So hätte das Mediamorphose-Modell etwa mit dem ebenso prominenten Production of Culture-Paradigma (entwickelt von Richard Peterson) kontrastiert werden können. ${ }^{12}$ Auch wenn Hemmings umfassende geschichtliche Rekonstruktion der Verlagskultur bzw. der Tonträgerindustrie und den damit zusammenhängenden Verwertungsstrukturen des Musikgeschäfts sehr anschaulich ausfällt, drängt sich bei diesem Kapitel erneut die Frage auf, mit welchen verschiedenen Me- thoden denn nun konkret die Musikökonomie und das Musikrecht untersucht werden können. Wo liegen die Vor- und Nachteile des Mediamorphose-Modells im Vergleich zu anderen, nicht näher genannten Analysezugriffen?

In Kapitel 9 »Globalisierung« blickt Hemming auf populäre Musik als transnationales Phänomen. In Anknüpfung an das vorangegangene Kapitel zur Musikökonomie wird das Genre 'Weltmusikı als Industrieprodukt diskutiert. Außerdem wird kritisch auf die Gefahren eines Eurozentrismus hingewiesen. Hemming zeichnet ein Porträt einer nicht machtfreien globalen Musikkultur in Anschluss an Theorien der Globalisierung (Arjun Appadurai) und des Postkolonialismus (Edward Said, Gayatri Chakravorty Spivak) und führt die dafür zentralen Begriffe `Othering eine gelungene Übersicht her, die Einblicke in diverse Forschungsansätze und -diskurse liefert. Hemming beschränkt sich auf einige konkrete und prominente Beispiele (der Indienbezug der Beatles, Paul Simons Graceland) und sieht von Exkursen ab.

Kapitel 10 »Geschichte und Geschichtsschreibung " stellt verschiedene Formen der Geschichtserzählung vor. Hemming unterteilt diese in "Leben und Werk", "Sozialgeschichte«, »Ereignisgeschichte versus Organismusmodell « und »spezielle Leitparadigmen « (461465). Einen Abschnitt zur Neuen Kulturgeschichte, jenem geschichtswissenschaftlichen Paradigma, das in den 1980er und 1990er Jahren aus der Sozialgeschichte hervorgegangen ist, sucht man allerdings vergebens. Gelungen sind Hemmings Ausführungen zum Forschungsdesiderat einer Popmusikgeschichte vor 1900 (465-472), da auch schon die bürgerliche Moderne seit Ende des 18. Jahrhunderts eine ausgeprägte Unterhaltungskultur kannte. Der Begriff des Populären hatte auch in dieser Zeit bereits Einzug in kulturelle Debatten und Diskurse über Musik und Kunst gehalten. Mit »Stockhausen - Ein Pionier des Techno? (473478) und "Kraftwerk - Pioniere des Techno?" (479-491) bietet Hemming aufs Neue zwei sehr raumfüllende Anwendungsbeispiele an, um das "Organismusmodell « auf die Probe zu stellen. Hemming entdeckt Spuren des Techno in der Arbeitsweise von Stockhausen und Kraftwerk, wobei er dafür argumentiert, dass die Entwicklung des Techno nicht exklusiv auf 
die "Keimzellen " Stockhausen und Kraftwerk zurückgeführt werden sollte. Das Kapitel schließt mit einem weiteren Anwendungsbeispiel zu "Schlager, Grand Prix und volkstümliche Musik im Fernsehen « (491-496), das indes viel zu kurz ausfällt. Hier soll Geschichtshermeneutik als Methode kritisch hinterfragt werden. Hemming plädiert dafür, geschichtlich jüngere Phänomene wie den Schlager auch mit Rückgriff auf ethnographische Methoden zu untersuchen. Doch wie genau dieser - eher vage vorgeschlagene - geschichtswissenschaftliche Zugang an die bisherige Forschung anschließen kann, erschließt sich nicht, zumal sich Hemming noch nicht einmal die Mühe macht, auf bereits vorgelegte historische und ethnologische Studien zum Schlager zu verweisen. ${ }^{13}$

Im letzten Kapitel 11 »Zur Definition populärer Musik« befasst sich Hemming mit einer Begriffsbestimmung von populärer Musik. Hemming schließt also mit einem Kapitel, welches den Gegenstand des Buches zu definieren oder einzugrenzen sucht. Wie erwähnt, drängt sich die Frage auf, warum dieses Kapitel nicht an den Anfang gestellt wurde. Denn bis hierhin bleibt in Hemmings Buch ungeklärt, was genau unter dem Begriff spopuläre Musikı zu subsumieren sei. Taggs »axiomatisches Dreiecksmodell « zur Unterscheidung von "Folk Music», »Art Music» und »Popular Music« wird vorgestellt, wobei der Autor kritisch anmerkt, dass eine "Auflistung essentieller Attribute" (505), wie sie Tagg unternimmt, ungeeignet sei, um populäre Musik als Gegenstand einzugrenzen. Kein so umfangreich erstellter Kriterienkatalog könne einem so komplexen kulturellen Gegenstand wie der populären Musik gerecht werden. Was aber nun genau als populäre Musik zu gelten hat, darauf legt sich Hemming in diesem Schlusskapitel nicht fest.

Insgesamt bleibt festzuhalten, dass Hemming mit Methoden der Erforschung populärer
Musik einen gewagten Vorstoß auf ein stark interdisziplinär geprägtes Feld unternimmt. Beeindruckend ist die Breite des Buches, das in seinen elf Kapiteln Einführungen in die diversen Themenfelder der Popmusikforschung bietet. Dennoch ist zu konstatieren, dass viele Aspekte, sofern sie nicht ganz fehlen, nur im Schnelldurchgang angerissen werden. Dass ein Methodenbuch nicht ohne Auslassungen auskommen kann und Schwerpunkte setzen muss, die der eigenen Expertise geschuldet sind, kann dem Autor nicht angelastet werden. Allerdings darf bemängelt werden, dass der Einblick in konkrete Themenfelder oft nicht dem aktuellen Forschungsstand entspricht. Jüngere Forschungsperspektiven der Popular Music Studies werden ausgeklammert und überhaupt nicht diskutiert (z. B. Sound Studies, Musicology of Record Production) und häufig wird auf Beispiele aus der Kunstmusik und Studien aus der Historischen Musikwissenschaft Bezug genommen, die auf populäre Musik nicht ohne weiteres übertragbar oder anwendbar sind. Es fehlt an Systematisierungen und kontrastierenden Gegenüberstellungen der Vor- und Nachteile verschiedener Methoden. Daneben ist das Layout des Buches zu bemängeln. Farblose und zum Teil unscharfe Grafiken und Bilder, zahlreiche Einschübe und eingefügte graue Kästen zerklüften das Erscheinungsbild und erschweren die Lesbarkeit des Buches.

Studierende und Forschende, die einen ersten Einstieg in das Feld der Popmusikforschung wünschen, können in Methoden der Erforschung populärer Musik somit lediglich einige wenige Eindrücke gewinnen. Wer sich eine kritisch aufgearbeitete Diskussion aktueller Entwicklungen und Debatten zu Methoden der Popmusikforschung erhofft, wird mit Hemmings selektiver Auswahl nur bedingt arbeiten können.

Steffen Just 


\section{Literatur}

Barthes, Roland (2013), „Die Rauheit der Stimme« [1977], in: ders., Der entgegenkommende und der stumpfe Sinn, Frankfurt a. M.: Suhrkamp, 269-278.

Brackett, David (1995), Interpreting Popular Music, Cambridge: Cambridge University Press.

Butler, Mark J. (2014), Playing with Something that Runs. Technology, Improvisation, and Composition in DJ and Laptop Performance, New York: Oxford University Press.

Elflein, Dietmar (2010), Schwermetallanalysen. Die musikalische Sprache des Heavy Metal, Bielefeld: transcript.

Hawkins, Stan (2002), Settling the Pop Score. Pop Texts and Identity Politics, Aldershot: Ashgate.

Heesch, Florian / Barbara Hornberger (Hg.) (2015), Rohe Beats, harte Sounds. Populäre Musik und Aggression, Hildesheim: Olm.

Helms, Dietrich / Thomas Phleps (Hg.) (2011), Black Box Pop. Analysen populärer Musik, Bielefeld: transcript.

Henriques, Julian (2011), Sonic Bodies. Reggae, Sound Systems, Performance Techniques, and Ways of Knowing, New York: Continuum.

Jarman-Ivens, Freya (Hg.) (2007), Oh Boy! Masculinities and Popular Music, New York: Routledge.

Jarman-Ivens, Freya (2011), Queer Voices. Technologies, Vocalities, and the Musical Flaw, New York: Palgrave Macmillan.

Kristeva, Julia (2010), Die Revolution der poetischen Sprache [1974], Frankfurt a. M.: Suhrkamp.

Mendívil, Julio (2008), Ein musikalisches Stück Heimat. Ethnologische Beobachtungen zum deutschen Schlager, Bielefeld: transcript.

Middleton, Richard (1990), Studying Popular Music, Buckingham (PA): Open University Press.

Moore, Allan F. (2012), Song Means. Interpreting and Analysing Recorded Popular Song, Farnham: Ashgate.

Müller, L. J. (2018), Sound und Sexismus. Geschlecht im Klang populärer Musik. Eine feministisch-musiktheoretische Annäherung, Hamburg: Marta Press.
Peterson, Richard A. (1989), „Five Constraints on the Production of Culture. Law, Technology, Market, Organizational Structure and Occupational Careers«, in: American Popular Music. Readings from the Popular Press, Bd. 1, hg. von Timothy Scheurer, Bowling Green: Bowling Green State University Press, 16-27.

Rappe, Michael (2010), Under Construction. Kontextbezogene Analyse afroamerikanischer Popmusik, Köln: Dohr.

Schröter, Jens / Axel Volmar (Hg.) (2013), Auditive Medienkulturen. Techniken des Hörens und Praktiken der Klanggestaltung, Bielefeld: transcript.

Tagg, Philip (1982), »Analysing Popular Music. Theory, Method and Practice», Popular Music 2, 37-67.

Tagg, Philip (2000), Kojak. 50 Seconds of Television Music. Toward the Analysis of Affect in Popular Music [1979], New York: Mass Media Music Scholars' Press.

Tagg, Philip (2001), Fernando the Flute. Analysis of Musical Meaning in an Abba Mega-Hit [1981], New York: Mass Media Music Scholars' Press.

Whiteley, Sheila (Hg.) (1997), Sexing the Groove. Popular Music and Gender, London: Routledge.

Whiteley, Sheila / Jennifer Rycenga (Hg.) (2006), Queering the Popular Pitch, New York: Routledge.

Wicke, Peter (2003), „Popmusik in der Analyse«, Acta Musicologica 75/1, 107-126.

Wicke, Peter (2008), „Wenn der Zeitgeist singt. Warum Schlager klingen, wie sie klingen«, in: Melodien für Millionen. Das Jahrhundert des Schlagers, hg. von Stiftung Haus der Geschichte der Bundesrepublik Deutschland, Bielefeld: Kerber, 14-21.

Zagorski-Thomas, Simon (2014), The Musicology of Record Production, Cambridge: Cambridge University Press.

Zak, Albin (2001), The Poetics of Rock: Cutting Tracks, Making Records, Berkeley: University of California Press. 
Just, Steffen (2018): Jan Hemming, Methoden der Erforschung populärer Musik (Systematische Musikwissenschaft), Wiesbaden: Springer 2016, https://doi.org/10.1007/978-3-658-11496-1. ZGMTH 15/2, 231-238. https://doi.org/10.31751/976

(C) 2018 Steffen Just (steffen.just@hu-berlin.de)

Dieser Text erscheint im Open Access und ist lizenziert unter einer Creative Commons Namensnennung 4.0 International Lizenz.

This is an open access article licensed under a

Creative Commons Attribution 4.0 International License.

eingereicht / submitted: 30/08/2018

angenommen / accepted: 31/08/2018

veröffentlicht / first published: 18/12/2018

zuletzt geändert / last updated: 18/12/2018 